Invited Paper

\title{
AUTHENTICATION, PRIVACY, SECURITY CAN EXPLOIT BRAINWAVE BY BIOMARKER
}

\author{
Jeffrey Jenkins ${ }^{1}$, Charles Sweet ${ }^{1,2}$, James Sweet ${ }^{2}$, Steven Noel ${ }^{1}$, Harold $\mathrm{Szu}^{3}$ \\ ${ }^{\prime}$ George Mason University \\ ${ }^{2}$ Loft Mind, Inc. \\ ${ }^{3}$ Catholic University of America
}

\begin{abstract}
We seek to augment the current Common Access Control (CAC) card and Personal Identification Number (PIN) verification systems with an additional layer of classified access biometrics. Among proven devices such as fingerprint readers and cameras that can sense the human eye's iris pattern, we introduced a number of users to a sequence of 'grandmother images', or emotionally evoked stimuliresponse images from other users, as well as one of their own, for the purpose of authentication. We performed testing and evaluation of the Authenticity Privacy and Security (APS) brainwave biometrics, similar to the internal organ of the human eye's iris which cannot easily be altered. 'Aha' recognition through stimulus-response habituation can serve as a biomarker, similar to keystroke dynamics analysis for inter and intra key fluctuation time of a memorized PIN number (FIST). Using a non-tethered Electroencephalogram (EEG) wireless smartphone/pc monitor interface, we explore the appropriate stimuli-response biomarker present in DTAB low frequency group waves. Prior to login, the user is shown a series of images on a computer display. They have been primed to click their mouse when the image is presented. DTAB waves are collected with a wireless EEG and are sent via Smartphone to a cloud based processing infrastructure. There, we measure fluctuations in DTAB waves from a wireless, non-tethered, single node EEG device between the Personal Graphic Image Number (PGIN) stimulus image and the response time from an individual's mental performance baseline. We hope Higher Order Statistics (HOS) in typing and PGIN can reveal intrinsic personal traits/habits and increase EEG specificity through stimulus-response neuro-biomarkers. Towards that goal, we describe an infrastructure that supports distributed verification for webbased EEG authentication. The performance of machine learning on the relative Power Spectral Density EEG data may uncover features required for subsequent access to web or media content. Our approach provides a scalable framework wrapped into a robust NeuroInformatics toolkit, viable for use in the Biomedical and mental health communities, as well as numerous consumer applications.
\end{abstract}

Keywords: Biomarkers, Physiology, Neural Information Processing, Biomedical Wellness, Scalable Infrastructures, Smart Sensor Networks.

\section{1 - INTRODUCTION}

In this paper, we describe how to augment current digital User Access Control (UAC), such as CAC cards, and verification systems like a memorized PIN, with an additional layer of security using non-tethered Electroencephalogram (EEG) devices. The aim of hardening UAC verification is not only beneficial for soft biometric enhancement of computer terminal cyber security, but may be valuable to exploit cognitive biomarkers to detect a transition from wellness to illness. Towards this goal, we describe an infrastructure that supports distributed verification for web-based EEG authentication and some of the performance metrics required for access to web content. A user attempting to gain access to digital content protected by EEG-UAC soft biometrics shall perform a series of sensory evoked memory recall perception tests. Among proven devices such as fingerprint readers and cameras that can sense the human eye's iris pattern, we introduced a number of users to a sequence of 'grandmother images', or emotionally evoked stimuli-response images from a number of other users, as well as a single image chosen by the user requesting access for the purpose of authentication. We performed testing and evaluation of the Authenticity Privacy and Security (APS) brainwave biometrics, similar to the internal organ of the human "Iris" which cannot easily be altered. 'Aha' recognition through stimulus-response habituation can serve as a biomarker, similar to keystroke dynamics analysis for inter and intra key fluctuation time of a memorized PIN number (FIST). A user's response to their chosen image can be entry of a PIN number, or simply a mouse click, which can combine two bio-physical data sources into a biomarker. To achieve this biometric APS enhancement, per restricted entry point the package will cost: touch screen display device $\sim \$ 100$, a COTS wireless EEG headset $\sim \$ 200,+$ know how. We can implement RSA coding to encrypt an unknown (to the user and admin) alphanumeric PIN number which comes explicitly from the experimental design and hardware implementation (testable for SuperComputer PC decryption testing). Using a single node COTS EEG, we provide a backup reading of DTAB fluctuation before and after the discovery of PGIN

Independent Component Analyses, Compressive Sampling, Wavelets, Neural Net, Biosystems,

and Nanoengineering XII, edited by Harold H. Szu, Liyi Dai, Proc. of SPIE Vol. 9118, 91180 U

(C) 2014 SPIE · CCC code: $0277-786 X / 14 / \$ 18 \cdot$ doi: 10.1117/12.2051323

Proc. of SPIE Vol. $911891180 U-1$ 
imagery. There must be a way to utilize this setup for Security, Interrogation, Information Assurance, and many more. We demonstrate this theory in a non-specific way in this paper, but can be used in the future for enhancement of secure systems.

Our $E E G \& f-E E G$ are implemented in a user-friendly manner, where the stimuli-responses could happen in a natural living environment with limited sensitivity, of which the specificity could be sharpened by noninvasive biomarkers. Note that the information of EEG is not so much present in the content of low frequency group brainwaves (DTAB $<30$ $\mathrm{Hz}$ in $8 \mathrm{~Hz}$ intervals); but rather in the spatiotemporal sensory stimuli correlation with a spatiotemporal response. In other words, the flow of neuronal ion currents collectively gave spatiotemporal information. For example, at a highly restricted gate entrance, traditional Common Access Card (CAC) and Personnel Identification Number (PIN) could use an absolutely irreplaceable authentication check provided with EEG head mount, key board, touch screen (a total cost about \$500 plus the know-how). We can tap into the secret individual emotional-IQ, loosely speaking, the Limbic system, the Amygdale, and the right of the side brain next to the Hippocampus, might be based on the visual /smell/ sensory stimuli of childhoodimprinted 'grandmother images'. We test the hypothesis of "grandmother cells" as a virtual biomarker by measuring the neuronal sensory responses using an $E E G$ head mount to achieve the specificity.

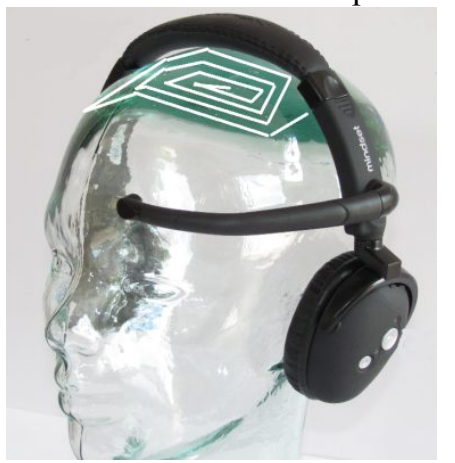

Fig. 1a - Single node head-mount showing 1-D Hilbert-Peano curve 'Spiral without origin' which provides EEG and f-EEG readout from single node. This measures the intrinsic brainwave 'organ' under stimulus response biomarker which provides individual uniqueness, or specificity.

The Common Access Card (CAC) is already associated with a picture ID, fingerprints, but could be further augmented with the human eye's iris surrounding the pupil (Sir John Daugman, U. Cambridge), or Brain Computer Interface (BCI) (BOD working group) inserted at the entrance gate control slot. If we choose BCI, we could expand the Personnel Identification Number (PIN) to include a Graphic component, using a touch screen with Personnel Graphic/Grandmother Identification Numbers (PGIN) provided by employees embedded in a fractal mosaic pattern of 100 grandmother pictures assembled randomly on a daily basis to defeat any memorization attempt of temporal placement. The number is the sequence of touches on the touch-screen's coordinate grid. We followed the FDA gold standard (double blind, negative control, in sufficient statistics) to test \& evaluate individual brainwave EEG \& f-EEG together with "typing/touching/ FIST" Kurtosis ( $4^{\text {th }}$ order cumulant) touching at one of the grandmother faces. We have actually applied the design to demonstrate successfully with ROC curve (PD 98\% vs. FAR 2\%, repeated with N-back of N=1,2,3 for fear of habituation) the PGIN experiments, in terms of specificity of BCI typing/touching FIST evoked by a PGIN (grandmother) picture as the intrinsic e-IQ stimulus and the internal organ responses as EEG and f-EEG. Concurrently, KAIST has attempted the interesting notion of brainwaves as fingerprints (S-Y Lee, Eun-soo Jung, Proc. SPIE Baltimore 2014).
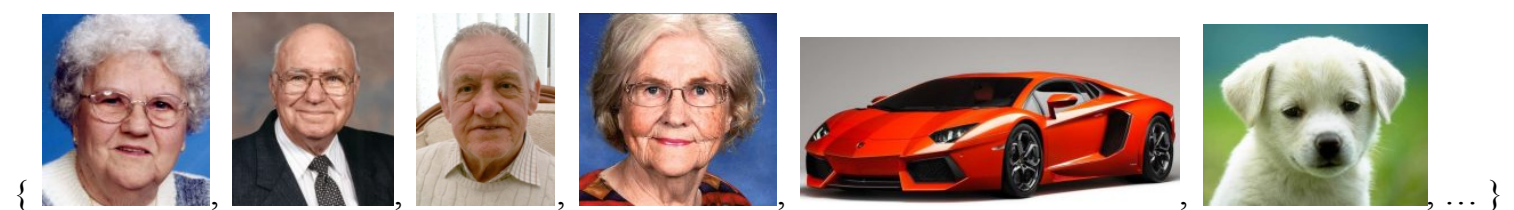

Fig. $1 \mathrm{~b}$ - An exemplar set of at least 6 images per individual, i.e. Grandparents faces, pet, car, etc.

(i) Construct a set of at least 6 images per individual, i.e. Grandparents faces, pet, car, etc. These images should be collected from every employee upon enrollment, for an IT Administrator to ensure security and admissible entry for a subset of all employees to a restricted site. (ii) At the entry point, a smartcard reader, upon card insertion, a timer begins T0, and discovery of PGIN image will cause timestamp T1, T2, .. to generate 'FIST' for the user via touch screen display for verification. PGIN testing can provide specificity through Higher Order Statistic (HOS) Kurtosis. In common security coding, we often wonder - how many words (most frequent word count statistic) characterize a person's habits? This 
frequency count implies education level as well as other descriptors of an individual, where a pair of words is a correlation. Shakespeare uses 4 words in a certain structure, it characterizes his writing and can be recognized among more common words. HOS may tell you something about personal traits/habits/characteristics, but you typically need thousands of data points. The typing FIST type varies from person to person - a user friendly, personalized APS method that is normalized by the intrinsic statistical representation of the PGIN. We can predict the next FIST fluctuation value in HOS kurtosis, and find percent change from the real statistical value to update our ANN learning matrix to be described later.

$$
\begin{gathered}
X_{i+1}=x_{i}^{0}+\eta\left(X_{i}-x_{i}^{0}\right) \frac{\partial K\left(X_{i}\right)}{\partial x_{i}^{0}}=\left(\vec{W}, \overrightarrow{X_{l}}\right)=a+b X_{i}+c X_{i}^{2}+d X_{i}^{3} \\
\frac{\widetilde{\mathrm{X}}_{m+1}-X_{m+1}}{\widetilde{\mathrm{X}}_{m+1}} \%>\varepsilon^{(5)} \%
\end{gathered}
$$

There is a bounded time for each user that will be used to reject/accept FIST values, and upon rejection or suspicion of the user recognizing a pattern in the location of their image they should be imaged by a camera at the entry point to provide a secondary check for authorization. (iii) Form a mosaic pattern around fractally distributed discrete grid coordinate for RSA coding to be updated every $n$ days, tbd by penetration testing.

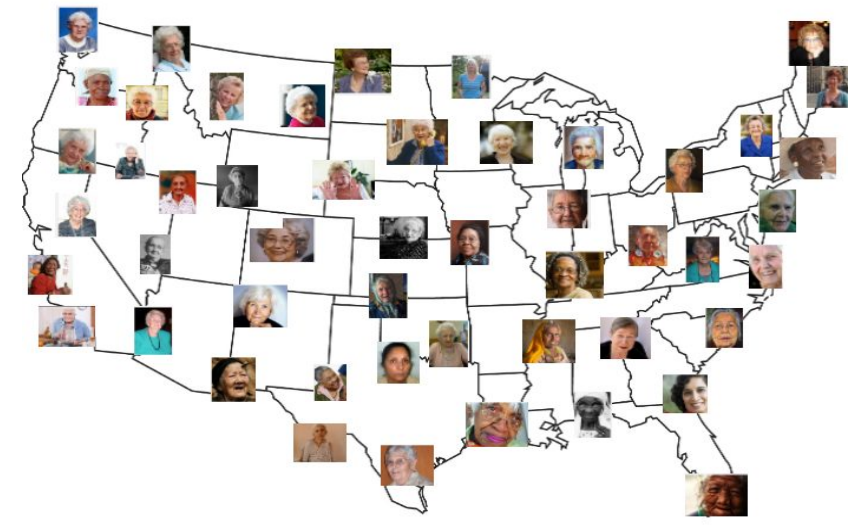

Fig. 1c - A mosaic pattern around fractally distributed discrete grid coordinate for RSA coding.

(iv) When the centroid of the image and touch location distance is minimized, a user has touched their PGIN image and we compare past FIST value predictions to current to authenticate user. Our approach has been shown to be effective after roughly two weeks worth of login attempts, rather than the $>300$ trials required for habituation for 6 digit PIN number FIST.

EEG signals have been harnessed to enable individuals to communicate using a presentation/selection paradigm based on the P300 response. In this manner, an individual could conceivably enter a shared key provided by input selected from the user. Such an approach is little more than a username/password system with a different user interface device (essentially providing no security improvement). The presentation/selection paradigm is very slow for letter selection, and though tolerable for word selection, potential adversaries may have an easier time provided a dictionary to draw potential passwords from. This would make it much more difficult to enter passwords for all but the most debilitated individual, and would potentially diminish the security of passwords by providing a set list of candidates. We instead propose a nontraditional methodology for achieving real-time authentication of an individual through a uniquely established baseline. Thus, modern mobile devices are a natural fit for pairing EEG devices with a soft-biometric authentication service. EEG data collection equipment is small enough to be transported with an individual and consumers have already started to use biometric peripherals like Nike fit, Google glasses, FitBit, etc. Mobile devices are made for portability, and when coupled with the portable nature of cutting edge biometric equipment, an EEG would add value in any situation where network access is required; from cases such as an at-home web based medical resource, to entering a high security area with multimodal netted biometric identification systems.

Using a non-tethered Electroencephalogram (EEG) wireless smartphone/pc monitor interface, we uncover the 
appropriate stimuli-response in DTAB low frequency group waves. The STM information is not so much present in the in the low frequency content (DTAB $<30 \mathrm{~Hz}$ in $8 \mathrm{~Hz}$ intervals); rather the stimulus biomarkers and correlated responses. For example, individual brainwaves could serve as the Authentication, Privacy and Security (APS) biometrics that cannot be altered. As a security feature, we could display a composition of hundreds of random images which contain a number of specific images chosen by a user. The randomized fractal graphic collection may contain images similar to the individual's stimuli images [14]. An 'organization' requiring classified access will provide their own personalized images (Fig. 1). They form random compositions in a fractal mosaic pattern and are shown with one randomly placed personal emotional image by the users in parallel. While all the images were being displayed, we collected the user's wireless head mounted EEG output, which represents the relative amplitudes of individual EEG frequency bands (alpha, beta, gamma, theta, delta). To provide an intrinsic e-IQ emotional memory utilization time, the user was instructed to click a mouse upon seeing one of their Grandmother images. We assert that the difference in time between seeing an image and clicking a mouse in response will statistically converge to an upper bound for a physical hand-eye coordination reaction [10]. Then, a Personalized Graphic/Grandmother Identifier Number (PGIN) stimulus-response test can established and executed upon attempted access of a computer system and serve as the APS stimulus biomarkers, then measure their FIST typing responses to differentiate many users. We begin by discussing the EEG and other brain monitoring devices, and current UAC systems. Then, we derive the recursive Higher Order Statistics (HOS) procedure to predict the next reaction fluctuation time. Results of single and multiple user classification ROC curves and success/failed classification is demonstrated. We then describe applications of this work to enhance security of web-based UAC, as well as interfacing with control of other electronic devices to enhance Participatory, Personalized, Preemptive, and Preventative (4-Ps, coined by Dr. Elias Zerhouni of the NIH) biomedical wellness and biometric systems.

\section{2 - MODERN MEDICAL TOOLS AND APPLICATIONS FOR BRAIN IMAGING}

Although this technology is still relatively young, doctors have suggested that EEG and $\boldsymbol{f}$-EEG (Szu et. al, SPIE 2014) may be a practical device for strengthening mental health, as well as education [5]. Psychiatrists have long trained the brain's pre-frontal cortex to fight against acute conditions like anxiety, post-traumatic stress and attention-deficit disorder. Practices like meditation, exercise and cognitive therapy have shown comparable short-term efficacy to medication for these disorders [1]. If coupled with other non-contact sensors, such as the Microsoft Kinect, keyboard, or an acoustic sensor, more specific information could be extracted from players. This could potentially aid physics-based gaming engines in responding quicker to a random movement from users. A signal from a particular region of the brain is typically involved in performing a specific action. Thus, refining the software and hardware which mobile EEG systems use is important in order to demonstrate how these devices can impact the way humans interact with and in a virtual environment. The entire gaming industry could be greatly impacted if this device were to be incorporated into mainstream gaming platforms. Players could not only be improving cognitive health by using their mind in new ways, but would benefit from a greater level of immersion using this device in concert with other sensing devices that provide input into games. An interesting property arising out of this technology which should be investigated is how brain wave analysis could aid in smartly extracting from other sensors. For instance, if you thought to move your leg, a characteristic brain wave pattern would be sensed before it is physically possible to actually move your leg. This could help other sensing devices to anticipate data arising out of disjoint sensing elements to feed into the virtual representation presented through Augmented Reality (AR).

The MagnetoEncephaloGram (MEG) is a brain dynamics measurement tool which requires the patient to sit under a sensor (which resembles a large salon hairdryer) that emits a high resolution magnetic field. The wired ElectroEncephaloGram (EEG) has been used for years to understand activity in the brain by measuring Local Field Potential (LFP) adjacent to sensing nodes. Functional Magnetic Resonance Imaging (f-MRI) is used to track the Blood Oxygen Level Dependency (BOLD) signal traveling through the head, but often suffers from low frame-rate. CAT and PET scanners are both similar devices, a large number of radial slice images are taken around the head and must be put back together via registration to the patient - an often difficult process. These non-invasive measurements utilize scalp recordings of brain field potentials known as Electroencephalogram (EEG) and Magneto-encephalogram (MEG). The parallel ion currents through collective striated layers of fan-in parallel input dendrite trees are proceed through neuronal threshold hillocks as fan-out axonal ion currents are the basis of EEG by means of Frequency Modulation (FM) for Far 
Field Communication (Ampere's law) or $M E G$ by means of Amplitude Modulations (AM) for Near Field Communication (Faraday induction law). It is common to our general telecom know-how about FM to be the basis for robust zero-crossing for resilient Long Distance Communication, and similarly FM may be across every brain functional regions. Home Alone Seniors (Has) and Juniors all require mental healthcare. We found the Biomedical Wellness Baseline: ubiquitous fat-tail distributions of PSD for healthy brains operating at $37^{\circ} \mathrm{C}=(\mathrm{SNR}) \times(1 / 40) \mathrm{eV}$, in outdoor environments $\left(27^{\circ} \mathrm{C}\right.$ : $\mathrm{SNR} \sim(310 / 300)$ [12][11]. The departure might implicate stress, fever, injury in sports and trip-over fall's, or numerous sleepwalks and the onset of dementia or Alzheimer's that could be discovered early by care-givers [15].

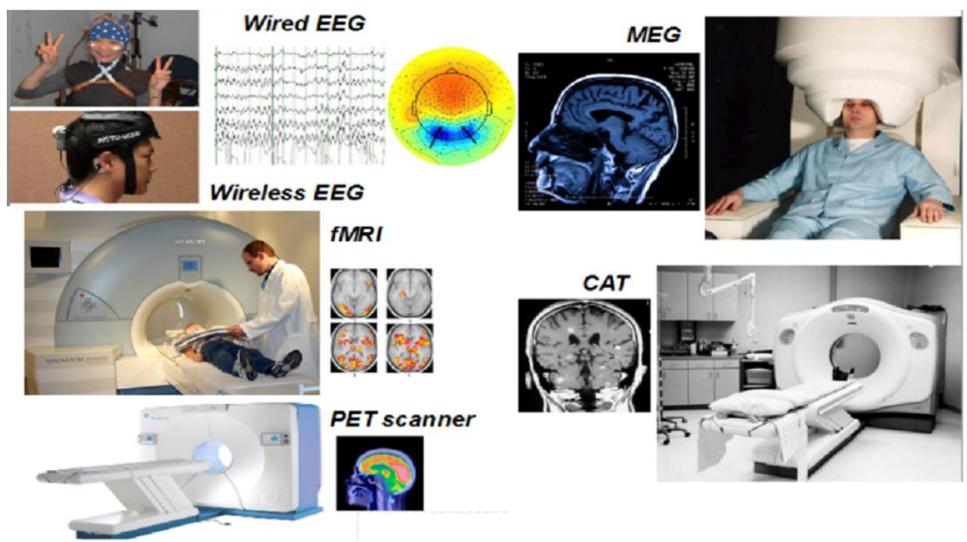

Fig. 2 - Examples of modern imaging devices, both invasive (active imaging inside of the body) and non-invasive (passive imaging of biofeedback), used in brain research.

Functional-Neuro-imaging usually involves some invasive contrast agents or molecular biomarkers: (a) $\boldsymbol{f}$ Magnetic Resonance Imaging (f-MRI) measures the Blood Oxygen Level Dependency (BOLD).Either way, it has been demonstrated that low-frequency EEG signals hold important information about an individual's 'brain state'. This 'brain state' can be a combination of conscious or subconscious brain dynamics - and it is often difficult to de-conflict internal dynamics that are initiated by different stimuli [7]. One such example if this state can be observed via the interaction between the Ventromedial Prefrontal Cortex (VPC) and the Amygdala. The VPC is responsible for inhibiting emotional responses arising from the Amygdala during fear suppression tasks; and this behavior is evident in most sensors having high spatiotemporal resolution. Other mechanisms of brain response can be coupled to visible measurements of pupil width. The increase in diameter pupil has been shown to be proportional to increases in cognitive load of the Short-Term Memory (STM) in the frontal cortex (Kahneman, Thinking Fast and Slow). In our case, we wish to measure the "AHA" moment of recognition memory through a visual priming calibration exercise. We will show that even a single dry node on a Wireless EEG can capture meaningful information from a stimulus-response test through the DTAB wave bands that are collected in real-time. This gives rise to the possibility of embedded microcircuits which perform a suite of behavioral and state classification procedures on real time data to leverage pools of data from worldwide contributors in our cloudbased push-pull web infrastructure. Standard DTAB waves were used to demonstrate the performance of translational Machine Learning (ML) algorithms on assessing the reliability of real-time bio-sensor data. Some intuition we gleaned from the data is that mental priming habituation of a pass-image takes roughly 20 sessions for different users. It appears that the statistical characteristics of DTAB data occurring before, during, and after, have very different properties and appear to be unique to each person. There are also other combinations of candidate features, but the real causal chain between a spike in one PSD band versus another could ultimately exist in the data.

The future of brain research is extremely promising due largely to advances in sensing and imaging technology. In the past, studying the electrical activity of neurons required electrodes to be inserted directly into the neuron. Electrode measurement is for high precision, single nerve electric potential (EP) over time, and experiments typically require numerous well placed electrodes to understand how larger collections of connected nerves behave. This methodology was adopted by Nobel laureates David Hubel and Torstien Wiesel, who discovered that the retinal ganglion cells of a cat responded directly to oriented edges [2]. By utilizing a time series of measurements, they derived a function which modeled the ganglion response mechanism; analogous to a Mexican hat wavelet used in image de-nosing and compression [12]. 
Imaging technology has revolutionized the way medical research is performed; devices can now measure the behavior of cellular mechanisms and functions with invasive and non-invasive methods.

\section{3 -MOBILE BMW AND APS FOR GLOBAL TELEMEDICNE AND SECURE SYSTEMS}

Authentication is always a concern for any protected resource. While traditional usernames and PIN passwords are passable for common internet resources, multi-factor authentication is becoming more common in the commercial sector, often involving a trusted third-party. Corporations like Google and SalesForce provide software programs for mobile devices which boast auxiliary authentication, and are now becoming widely available. How can wireless EEG data be useful for BMW and APS? Technology is all around us and the internet is even more pervasive, so what is the unique opportunity that we have here? We all know that individuals may want to use technology at times when they probably shouldn't (i.e. distracted, fatigued, or drunk driving), but everybody's case is different whenever they may attempt.
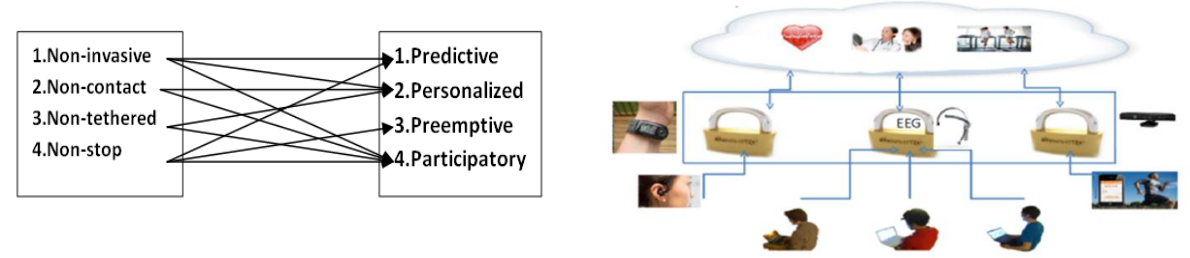

Fig. 3 - The future of networked Telemedicine must satisfy the 4-P and 4-non principles of BMW sensor systems. Individuals can practice proactive health habits while safeguarding private data with daily baseline 'login' checkups.

A brief review of public healthcare challenges can set the stage towards a strategy of meeting the mental and physical healthcare challenge for the aging global village. In addition to the ubiquitous public health insurance (Obamacare, etc.) programs, an equivalent Bill of Patient Right was given early by the former NIH director Dr. Zerhouni. In addition to the annual NIH federal budget of $\$ 37 \mathrm{~B}$ for 37 Institutes (one per human science components), Zerhouni has provided an additional $\$ 2 \mathrm{~B}$ budget for the translational R/D "from benches to the bedside" to support patients with the 4P principles, i.e. private, personalized, pre-emptive, and participatory, to establish distributed points of care (POC), thereby extending the local community hospital to households, becoming a "houspital." We shall also follow ONR's vision who offered an informal survey and concluded with a VA desire of the 4 user-friendly practices (4 non's = noninvasive, non-contact, non-tethered, and none-stop to receive treatments) as a fundamental biomedical wellness (BMW) device design principle. According to both the 4-P \& 4-Non's and the FDA Gold Standard to be harmless to patients, it is not entirely unthinkable to equip HAS with affordable and effective instrumentation [19]. We can take individual daily brain activity with a user-friendly wireless head mount, e.g. baseball hat, measuring both the averaged and the fluctuating pair correlation behaviors using powerful computational platform of Smartphone. We statistically refer to the $1^{\text {st }}$ order means as the EEG, \& the $2^{\text {nd }}$ order co-variances as a functional-EEG $(f-E E G)$.

Below, we investigate results of traditional Machine Learning tools and techniques and investigate if there are features buried in the data. Then using these features we build a classifier and iteratively refine the data representation until we have a high degree of certainty that 'you are who you say you are'. We know that building personalized classifiers for millions of users is do-able, but may not be sufficient in providing a concrete visual representation that is useful to facilitate Medical pre-emptive, predictive, preventive and participatory discovery of onset of disorders. We can model the likelihood that an individual is indeed who they claim to be based on statistical fluctuation in DTAB brainwave signals in real-time response. This implies end-to-end signal encryption, necessitating a client-server model, having control of both client and server endpoints. With such a system, there are some considerations that end up driving the design of new underlying technology, if one is to trust the system as an authentication provider.

The EEG is an internal organ like the iris; due to the iris fold morphology, it is a biometric. Brainwave frequency response (gamma $100 \mathrm{~Hz}$, etc.) to stimulus response is difficult to extend to an individual. General s-r tests may not elicit a unique response unless we can tap into '/emotionally evoked' memory to seek uniqueness in the DTAB-G data. 


\section{4 - NON-TRADITIONAL BIO-FEEDBACK DATA MINING BASED ON PHYSIOLOGY}

We began with 3 users that participated in 25 sequential sessions, where the task was for each user to watch a series of 30 images and click the mouse upon seeing an image which they had chosen prior to the experiment. There was an inter-stimulus interval of 2 seconds and an intra-stimulus interval of 1 second. The order of images was different each session, but the presentation order was the same for each user given the session number. The total 30 image set duration was fixed for all sessions too, while the location index of a user's image within a session was randomly generated and normalized for all users. Our implementation test-bed was fostered by a Brain Computer Interface (BCI) data collection/visualization web service (www.loftmind.com) maintained by some of the authors. We used Matlab for initial statistical analysis of raw DTAB values and visualizing session output. These algorithms were then implemented on our online test-bed for real-time scalability analysis.
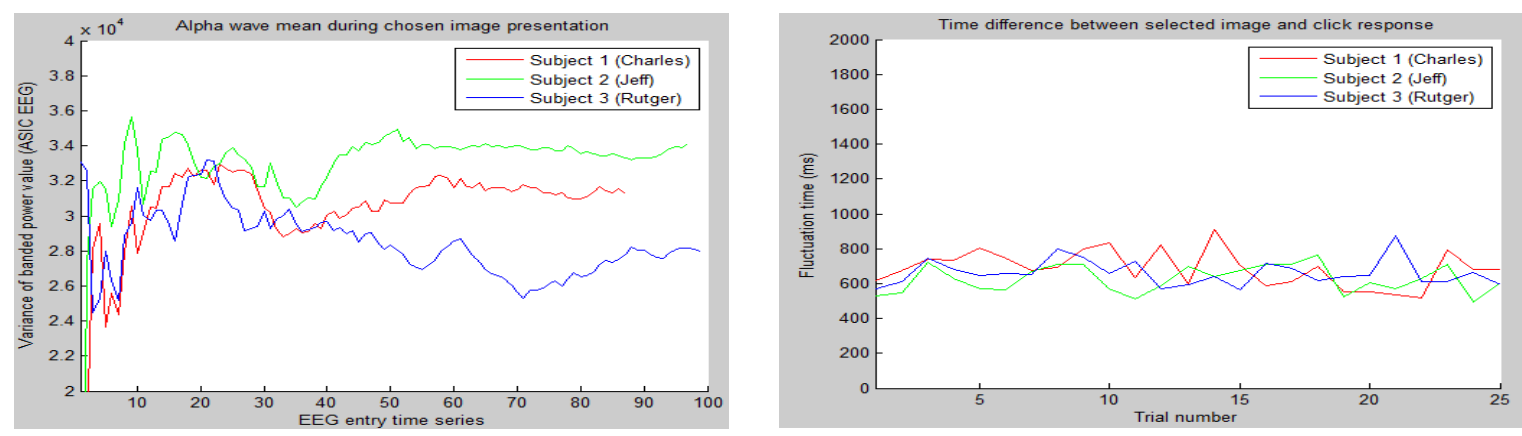

Fig. 4 - (a) The mean of the alpha wave labeled as 'in' indicates clear separation of users and also hints at a link between habituation and low long-run fluctuation from trend alpha wave. (b) Time difference between presentation of PGIN in sequence and subsequent click response time (shown for all users over all trials) is invariant to user and trial number.

The recursive mean formula is a simple, yet powerful tool for real time anomaly detection and prediction. For a data vector having $\mathrm{N}$ elements, the mean at time $\mathrm{t}$ is calculated in the typical fashion. However, when the next data point arrives, we only need to keep the previous mean and the number of values counted so far, and the next data point to determine the next mean value [10]. A recursive learning self organization map can do Higher Order Statistics (HOS) analysis, replacing the traditional batch mode average with a sequential associative memory update of a small orthogonal amount for the stability of change detection and habituation knowledge discovery [3]. This habituation has been demonstrated by some of the authors as the change in kurtosis of bio-sensor data over time for a particular user. Kurtosis is the invariant 4 th statistical moment which has a unique property. When Kurtosis has value of 0 , it is indicative of a Gaussian nature to the data - randomness and little statistical information. Otherwise, we assume stationarity - and measure statistical habituation through a single node placed on the ventromedial prefrontal cortex (Brodman's Area 10).

A common pitfall of EEG data exploration is assuming stationarity. EEG data conceals a well-established baseline drift, on top of reactions to stimuli from other mental processes. On top of that, noise reduction by averaging is only applicable in situations where the noise can be demonstrated to be random, or having a Kurtosis $\mathrm{K}=0$. This may not the case in EEG data, as some device specific, spatially correlated fluctuation noise could arise from the heat transfer of coincident incoherent waves originating in an unknown combination of internal brain regions. We seek stationarity by defining the time-windowed mean of a single electrode $\boldsymbol{\mu}-\boldsymbol{E} \boldsymbol{E}$ and the electrode-pair correlation of fluctuation functions $\boldsymbol{f}-\boldsymbol{E} E \boldsymbol{G}$ :

We shall compute the universal spatial correlation of Electroencephalogram (F-EEG) due to the thermal fluctuation effect. We decompose any measurement into ensemble-average and fluctuation as follows:

$$
\begin{aligned}
\boldsymbol{e}(\boldsymbol{x}, t)= & <\boldsymbol{e}(\boldsymbol{x}, t)>+\boldsymbol{e}(\boldsymbol{x}, t)-<\boldsymbol{e}(\boldsymbol{x}, t)>\equiv \overline{\boldsymbol{e}}(\boldsymbol{x}, t)+\tilde{\boldsymbol{e}}(\boldsymbol{x}, t) \\
& <\tilde{\boldsymbol{e}}(\boldsymbol{x}, t)>=<\overline{\boldsymbol{e}}(\boldsymbol{x}, t) \tilde{\boldsymbol{e}}(\boldsymbol{x}, t)>=0
\end{aligned}
$$




$$
<\boldsymbol{e}(\boldsymbol{x}, t) \boldsymbol{e}\left(\boldsymbol{x}^{\prime}, t^{\prime}\right)>=<\tilde{\boldsymbol{e}}(\boldsymbol{x}, t) \tilde{\boldsymbol{e}}\left(\boldsymbol{x}^{\prime}, t^{\prime}\right)>
$$

The pair correlation function computation complexity for $\mathrm{N}$ electrodes is of the order $O\left(N^{3}\right)$. The slowdown is caused by a sequential operation of do loops of variable length separation $\boldsymbol{x}-\boldsymbol{x}^{\prime}$. For shift invariant stationary cases, we can simplify pair correlation function by means of Wiener-Khinchin-Einstein theorem using Fast Fourier Transform (FFT) parallel computing. We shall compute first by FFT of F-EEG: $\boldsymbol{E}(\boldsymbol{k}, \omega) \equiv F T\{\boldsymbol{e}(\boldsymbol{x}, t)\}$, i.e., at O(N $\log \mathrm{N})$

$$
\boldsymbol{E}(\boldsymbol{k}) \exp (-i \omega t)=\int_{=\infty}^{\infty} d x \exp (i \boldsymbol{k} \cdot \boldsymbol{x}-i \omega t) \boldsymbol{e}(\boldsymbol{x}, t)
$$

We define Power Spectral Density (PSD) as the ensemble average of square-modulus:

We will prove in the following WKE theorem:

$$
\operatorname{PSD}(\boldsymbol{k})=<|\boldsymbol{E}(\boldsymbol{k})|^{2}>.
$$

$$
F T_{\boldsymbol{k} \rightarrow \boldsymbol{x}}^{-1}\{P S D(\boldsymbol{k})\}=<e\left(x_{o}, t\right) e\left(x_{o}+\boldsymbol{x}, t\right)>
$$

According to the WKE theorem, we can save computational complexity, instead of computing pair correlation directly< $\tilde{e}(\boldsymbol{x}, t) \tilde{e}\left(\boldsymbol{x}^{\prime}, t\right)^{*}>$, at the computational complexity of $\mathrm{O}\left(\mathrm{N}^{3}\right)$ together with sequential control of moving window index.

THEOREM 1 Wiener Khinchin Einstein (WKE) Theorem at $\mathrm{O}(\mathrm{N} \log \mathrm{N})$ :

If and only if Fourier mode-mode are decoupled

$$
<\boldsymbol{E}(\boldsymbol{k}) \boldsymbol{E}\left(\boldsymbol{k}^{\prime}\right)^{*}>=<|\mathbf{E}(\mathbf{k})|^{2}>\delta\left(\mathbf{k}-\mathbf{k}^{\prime}\right)
$$

Then the pair correlation function is shift invariant stationary .

\section{PROOF:}

$$
\begin{gathered}
\text { If stationary }\left\langle\boldsymbol{e}\left(x^{\prime}\right) \cdot \boldsymbol{e}^{*}(\boldsymbol{x})>=<\boldsymbol{e}\left(\boldsymbol{x}_{\boldsymbol{o}}+\boldsymbol{x}\right) \cdot \boldsymbol{e}^{*}\left(\boldsymbol{x}_{\boldsymbol{o}}\right)>=\int_{-\infty}^{\infty} d \boldsymbol{k} \int_{-\infty}^{\infty} \mathrm{d} \mathbf{k}^{\prime} \exp \left(i k\left(\boldsymbol{x}_{\boldsymbol{o}}+\boldsymbol{x}\right) \exp \left(-i \boldsymbol{k}^{\prime} \cdot \boldsymbol{x}_{\boldsymbol{o}}\right)<\boldsymbol{E}(\boldsymbol{k}) \cdot \boldsymbol{E}\left(\boldsymbol{k}^{\prime}\right)^{*}>\right.\right. \\
=\int_{-\infty}^{\infty} d \boldsymbol{k} \operatorname{PSD}(\boldsymbol{k}) \exp (\mathrm{ik} \cdot \mathbf{x})=F T\{\operatorname{PSD}(\boldsymbol{k})\} ;
\end{gathered}
$$

Then, the inverse Fourier Transform yields directly

$$
\boldsymbol{F T}_{\boldsymbol{k} \rightarrow x}^{-1}\{P S D(\boldsymbol{k})\}=<\tilde{e}\left(\boldsymbol{x}_{\boldsymbol{o}}, t\right) \tilde{e}\left(\boldsymbol{x}_{\boldsymbol{o}}+\boldsymbol{x}, t\right)^{*}>
$$

Conversely, if mode-mode is decoupled, then pair correlation is shift invariant stationary.

Once we know the data ascribes to this property (3a) we derive a recursive update formula to provide real-time anomaly detection to pre-process and reduce data storage of past noisy EEG data. We recursively compute H.O.S. of temporal vector inputs from stimulus response tests. One such test is as follows -3 images will be presented in an otherwise random sequence of images, and whenever one of the 3 images is shown, the user is primed to enter a PIN number. From past logins, we have collected data for each 'Air time' difference between keystrokes for a number of users. Then, we can use stimulus-response as a trigger and additional data point for real-time secure systems. To begin, we take the batch mode statistics of an individual stimulus response time prior to FIST dynamics in order to keep building accumulative statistics moments as follows. We define a uniform weighted batched average of our data set to be the angular brackets

$$
\left\langle x_{j}^{m}\right\rangle_{N}=\frac{1}{N} \sum_{j=1}^{N} x_{j}^{m}
$$

We prove a useful Lemma of Recursive Averages to any m-th moments

$$
\left\langle x_{j}^{m}\right\rangle_{N}=\frac{N}{N+1} \sum_{j=1}^{N}\left\langle x_{j}^{m}\right\rangle_{N}+\frac{1}{N+1} X_{N+1}^{m}
$$

Then, we can build in order the mean, variance, skewness, and kurtosis sequentially. At the current time with $\mathrm{N}$ observations, the mean

$$
\mu_{N}=\frac{1}{N} \sum_{j=1}^{N} x_{j} \equiv\left\langle x_{j}\right\rangle_{N}
$$

can update the next mean value by the following extension of (1), (2)

$$
\mu_{N+1}=\frac{N}{N+1}\left\langle x_{j}\right\rangle_{N}+\frac{1}{N+1} X_{N+1}
$$

The percent change between sequentially captured time difference data can be computed

$$
\frac{\mu_{N+1}-\mu_{N}}{\mu_{N+1}} \%>\varepsilon^{(1)} \%
$$

Similarly, the variance can be computed and updated

$$
\begin{gathered}
\sigma_{N}=\left\langle\left(x_{j}-\mu_{N}\right)^{2}\right\rangle_{N}=\left\langle x_{j}^{2}\right\rangle_{N}-\mu_{N}^{2} \\
\sigma_{N+1}+\mu_{N+1}^{2}=\left\langle x_{j}^{2}\right\rangle_{N+1}-\frac{N}{N+1}\left(\sigma_{N}+\mu_{N}^{2}\right)+\frac{1}{N+1} X_{N+1}^{2}
\end{gathered}
$$


with a percent fluctuation

$$
\frac{\sigma_{N+1}-\sigma_{N}}{\sigma_{N+1}} \%>\varepsilon^{(2)} \%
$$

Skewness is defined as

with an update formula of

$$
\gamma_{N}=\left\langle\left(x_{j}-\mu_{N}\right)^{3}\right\rangle_{N}=\left\langle x_{j}^{3}\right\rangle_{N}-\mu_{N}^{3}
$$

$$
\begin{gathered}
\gamma_{N+1}+3 \sigma_{N+1} \mu_{N+1}-\mu_{N+1}^{3}=\left\langle x_{j}^{3}\right\rangle_{N+1}=\frac{N}{N+1}\left(\gamma_{N}+3 \sigma_{N}+\mu_{N}^{3}\right)+\frac{1}{N+1} X_{N+1}^{3} \\
\frac{\gamma_{N+1}-\gamma_{N}}{\gamma_{N+1}} \%>\varepsilon^{(3)} \%
\end{gathered}
$$

Kurtosis is defined below,

$$
K_{N}=\left\langle\left(x_{j}-\mu_{N}\right)^{4}\right\rangle_{N}-3 \sigma_{N}^{2}=\left\langle\left(x_{j}\right)^{4}\right\rangle_{N}-R_{N} \quad(12) \quad R_{N}=\frac{N}{N+1} 4 \mu_{N} \gamma_{N}+6 \sigma_{N} \mu_{N}^{2}+\mu_{N}^{4}+3 \sigma_{N}^{2}
$$

and has a much more complicated update formula than the three lower moments

$$
K_{N+1}+R_{N+1}=\left\langle x_{j}^{4}\right\rangle_{N+1}=\frac{N}{N+1}\left\langle x_{j}^{4}\right\rangle_{N}+\frac{1}{N+1} X_{N+1}^{4}=\frac{N}{N+1}\left[K_{N+1}+R_{N+1}\right]+\frac{1}{N+1} X_{N+1}^{4}
$$

The fluctuation error is

$$
\frac{K_{N+1}-K_{N}}{K_{N+1}} \%>\varepsilon^{(4)} \%
$$

With this approach, we can predict the next difference in time between stimulus and response, just the same as interkeystroke duration at time $\mathrm{i}+1$ through a small change of the current $\mathrm{i}$ and the past summarized in the weight data. We define the data to be stationary around the mean value from all trials, $X_{i} \equiv x_{i}-\left\langle x_{i}\right\rangle ; x_{i} \equiv x\left(t_{i}\right) ; x_{i}^{0} \equiv x\left(t_{i}^{0}\right)$. Then, we predict the stimulus response fluctuation vector at trial i+1 from trial $\mathrm{i}$ data with the following (a-d from above eq's)

$$
X_{i+1}=x_{i}^{0}+\eta\left(X_{i}-x_{i}^{0}\right) \frac{\partial K\left(X_{i}\right)}{\partial x_{i}^{0}}=\left(\vec{W}, \overrightarrow{X_{l}}\right)=a+b X_{i}+c X_{i}^{2}+d X_{i}^{3} ; i=1,2,3, \ldots, m-1
$$

A user's uniquely defined weight matrix is

$$
\vec{W}=[\mathrm{a}, \mathrm{b}, \mathrm{c}, \mathrm{d}]^{T}
$$

Then, we establish our learning weight from the past data

$$
\left(\begin{array}{c}
\vec{X}_{2} \\
\vec{X}_{m}
\end{array}\right)=\left(\begin{array}{cccc}
1 & \vec{X}_{1} & \vec{X}_{1}^{2} & \vec{X}_{1}^{3} \\
\vdots & \ddots & & \vdots \\
1 & \vec{X}_{m-1} & \vec{X}_{m-1}^{2} & \vec{X}_{m-1}^{3}
\end{array}\right)\left(\begin{array}{l}
\vec{a} \\
\dddot{\vec{d}}
\end{array}\right)
$$

Where the weight matrix

$$
[\vec{W}]=\left(\begin{array}{c}
\vec{a} \\
\dddot{\vec{d}}
\end{array}\right)=[A]^{-1}\left(\begin{array}{c}
\vec{X}_{2} \\
\dddot{X}_{m}
\end{array}\right)
$$

We then arrive at the next time predicted result, to be compared with the true offset of time $X_{m+1}$

$$
\widetilde{\mathrm{X}}_{m+1}=a+b X_{m}+c X_{m}^{2}+d X_{m}^{3}
$$

and a departure in baseline statistics can be measured by a large value from the following equation:

$$
\frac{\widetilde{\mathrm{x}}_{m+1}-X_{m+1}}{\widetilde{\mathrm{x}}_{m+1}} \%>\varepsilon^{(5)} \%
$$

Below is a graphical representation of PIN number habituation in over 300 trials of data for 2 users of PIN number data which shows that the change of Kurtosis prediction of the next typing vanishes after habituation. We sought after 5
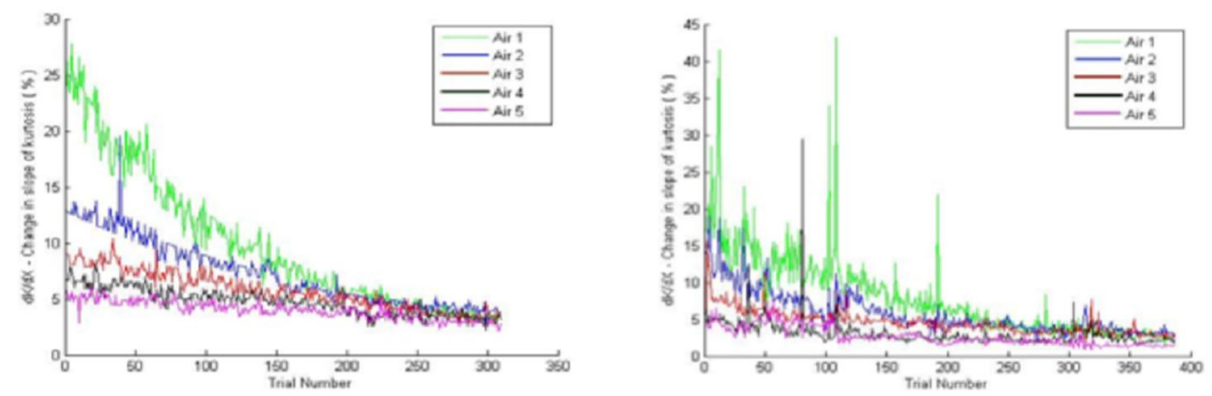

Fig. 5 - Change in Kurtosis for sequentially learned PIN numbers on standard keyboard - (a) User 1 typed PIN number with one hand every trial on pin pad, (b) User 2 typed PIN number with two hands every trial on horizontal number bar [16]. We wish to study the EEG signal concurrently with PGIN HOS. 
air times to find a stable kurtosis derivative prediction, and the ANN inverse of the Auto-Regressive prediction vs. real data as a prediction error.

From Fig 5, our initial work in soft-biometrics shows that the FIST of the sender (i.e. Morse code, WW2) indicated habituation in keyboard based bio-sensory data. This observation has led us to seek similar habituated behavior in the EEG. This reaction time contains within it a vector of separate time series which also revert to a mean value with low variance and near zero convergence of change in kurtosis. This is true with a high degree of accuracy when the percent change H.O.S. ANN USL algorithm is approximately 0 . We interpret this convergence as a habituation memory mechanism biomarker that has been detected by a few of the authors in other work [16]. Due to the striking visual representation of 'un-mined' data, we push forward with sophisticated machine learning tools to elucidate the invariant soft-biometric features within DTAB data.
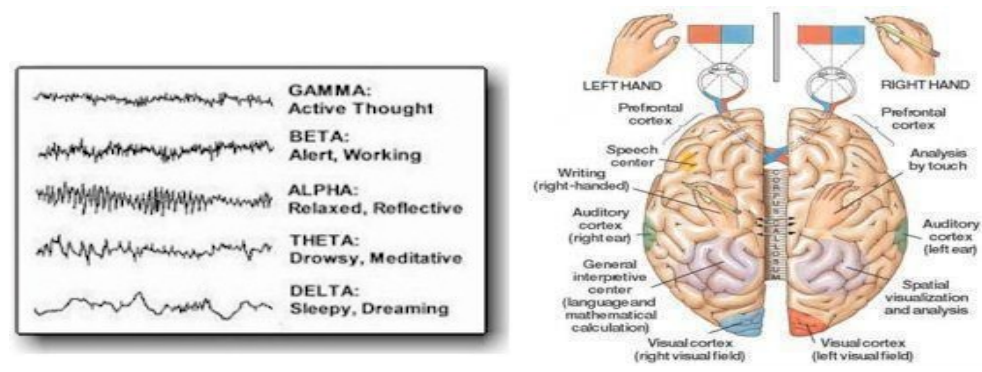

Fig.6 - (a) Typical DTAB $(G)$ waveforms, (b) functional brain areas, front-back/ side-side connectivity for sensory processing. We carried out the knowledge extraction process in WEKA, an open-source machine learning toolkit [13]. First we used the J-48 decision tree classifier to perform classification on the user label (trying to see if we could classify a user based on the data) and present the following graph of accuracy and ROC area for different attributes.

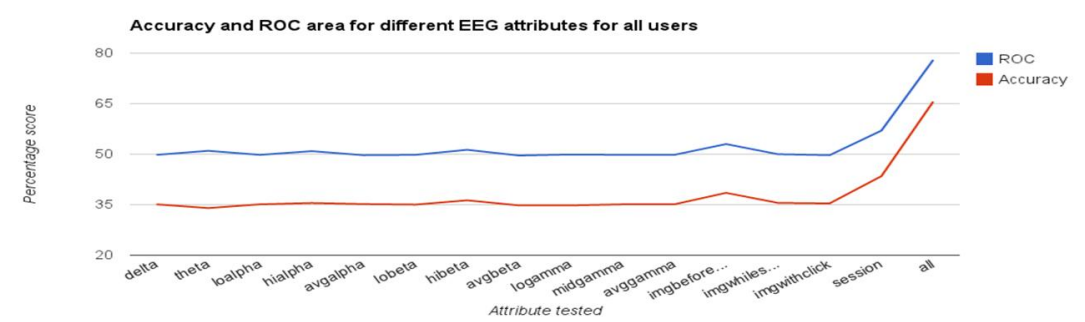

Fig. 7 -J-48 classification on unstructured/unfiltered session data shows no single attribute is good at single user classification.

In a user calibration session, time series data of user-specific EEG DTAB waves and user-specific PGIN presentation times are synched in order to label observations 'inside' or 'outside'. This label indicates whether entries are inside of the passimage presentation window (after image display time and before click response time), or outside of that window completely. We found this attribute was important for assessing if the EEG device was able to record enough data within the time window. We then performed J-48 classification for each user and discovered that user-specific habituation of DTAB waves was observed while analyzing session number versus the number of observations belonging to image within click, image while shown, and image before/after shown. 


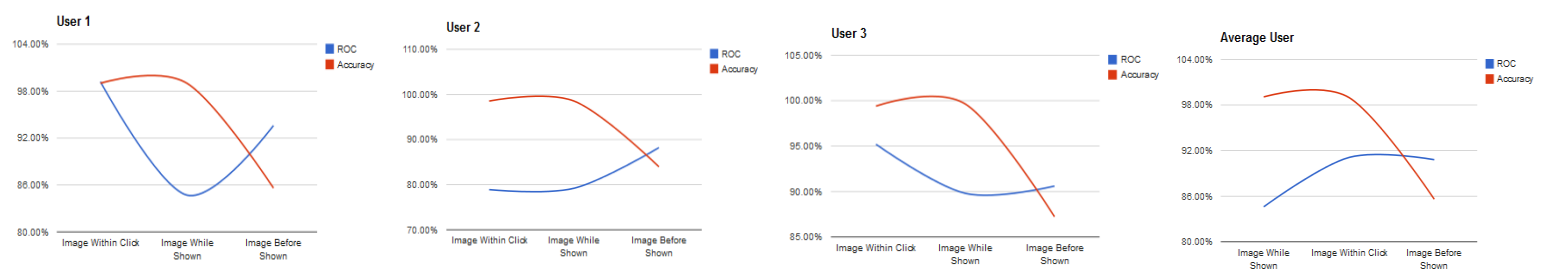

Fig. 8 - J-48 Classification results for single user attributes indicate similar features for individual user baseline difference classification. Image within click was the dominant feature encouraging candidate for fluctuation detection

Another interesting feature observation was, as the number of user sessions climbs, the 'image within click' label appears less frequently. This may be due to the Hippocampus (associative memory), which can prime muscle memory to effortlessly execute a physical action after recognizing contextual information. Less time to recognize then click would imply that less data points get recorded during this window. Intuitively this would indicate that the frequency of data points after a selected user's image can be useful as a classification feature on a per user basis - as this habituation seemed to appear for all users.
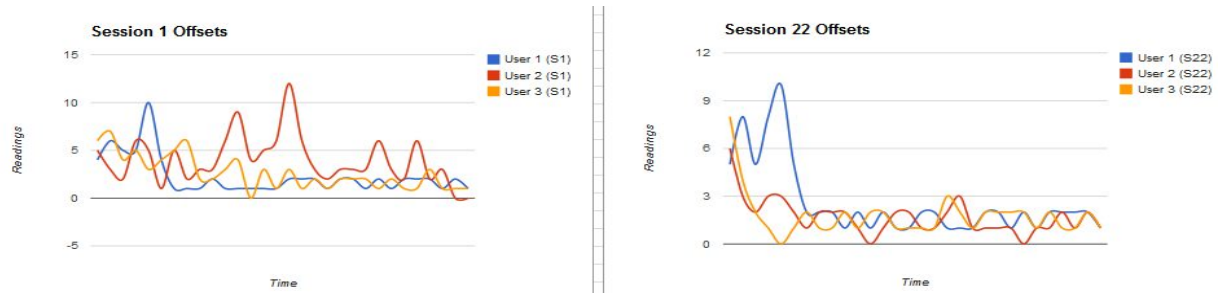

Fig. 9 - As time progressed in a session, the number of readings from the device is plotted on the y. (a) session 1 is the first run of the experiment, which shows less regularity in the number of readings recorded. Session 22 is one of the last runs of the experiment, indicating that habituation may have something to do with the number of observations recorded from the device.

The promising results of our study have inspired us to extend APS EEG with complexity based PGIN Recognition, which could provide visually degraded (at various levels of detail) perceptual information for individuals that do not have full or have degrading neural communication pathways from the front-to-back of the head [6]. An Associative Memory Neural Network approach will be developed to investigate how the foreground affects the background in imagery with varying complexities and quality for the same (memorized, primed PGIN) and different (somebody else's PGIN) imagery over time [18].

\section{5 - FUTURE WORK \& CONCLUSION}

We envision a human-centric approach to personalized Biomedical Wellness monitoring. A successful step towards truly bridging the patient and the caretaker relies on understanding the data from a number of sensing technologies - often in parallel. A slightly different sensing approach has removed the requirement for physical human movement to manipulate objects in 3-D. The head mounted device is an EEG (electro-encephalogram) which has sensors that translate brain waves into raw (signal + noise) digital information; useful for multiple commands if signals from certain brain regions can be properly de-mixed in real-time. This will provide new opportunities to game players as well as game developers. Current devices that are for sale can range from $\$ 150$ for lower density systems, and exceeding $\$ 2500$ for systems with more analysis and visualization features. An application for training effectiveness is also timely. Ensuring optimum performance in a workplace setting comes from balancing the personality traits and expectations of employees, who should learn to develop a balance between the anticipatory/intuitive emotional brain (e-Brain) and the focused and methodical left-hemisphere of the brain (1-Brain). To generate interest for such a study we could explore Economic thought/bargaining experiments as well as gathering $e$-Brain and $l$-brain experimental data from stimulus-response 
protocols. Through these types of experiments we can measure how well humans are able to perform collaborative decision-making though comparing a single person with two other persons using only one-side of the brain. For example, a game called 'MindHunter', which integrates real-time 3D graphics manipulation via consumer grade wireless EEG has been developed and requires players to focus very hard in order to fire a gun at an enemy. In this case, brainwave data and correct decisions would be kept as the double blind ground truth, while the control is the baseline performance of a single person with either $e$-Brain or $l$-Brain video information compared against two persons. Then, Higher order statistical Analysis can be performed to facilitate Triple Correlation through a set of dual measurements: i) movie-grade video stimuli, and ii) the responses include iris imaging and wireless EEG brainwaves data. This could capture the means by which individuals communicate a correct multiple choice answer - the process that we take to arrive at eureka and aha moments early in the collaborative decision-making process.

We have demonstrated an exemplar web-infrastructure for performing distributed testing of various protocols for Stimulus-Response experiment data representations through a computer terminal and mouse coupled with a low-density COTS EEG. Real-time monitoring was able to capture features of muscle memory habituation, measure of cognitive load, and baseline establishment within traditional DTAB waves. Then, we use PCA to combine and identify features, which proved useful for detecting, in an unsupervised way, any departure from individualized statistics. Our particular implementation and approach provides a viable alert mechanism for Mental Health specialist who can personally investigate the situation or immediately request help from a Medical professional [4]. This will help greatly in the future as we all must support the global aging village with technological advancements and increased participation. Our approach for detecting early signs of a transition from wellness to illness is general enough to handle real-time data fusion from additional psychological or physiological bio-feedback telemedicine devices.

\section{ACKNOWLEDGEMENTS}

We would like to thank Dr. Binh Tran, Dr. Keith Krapels, Susan Harkrider, Francois Lalonde, Steve Webster, and Jay Giedd, Rutger van Bergem, Justin Gates, and Shawn Gamble for helpful discussions and simulation support.

\section{REFERENCES}

[1] L. B. Jivanadham, A. K. M. M. Islam, Y. Katayama, S. Komaki, S. Baharun, "Cloud Cognitive Authenticator (CCA): A public cloud computing authentication mechanism," Informatics, Electronics \& Vision (ICIEV), vol. 1, no. 6, pp.17-18 May 2013

[2] D. Hubel, T. Wiesel, Receptive fields of single neurons in the cat's striate cortex, J. Physiol. (I959) I48, 574-591

[3] H. Szu, J. Jenkins, C. Hsu, S. Goehl, L. Miao, M. Cader, and D. Benachenhou, Digging for Knowledge, in Quantum Information and Computation VII, 2009, vol. 7343, pp. 734304-734317.

[4] Scott Makeig, Tzyy-Ping Jung, Terrence Sejnowski, Using feed forward neural networks to monitor alertness from changes in FEEG correlation and coherence," Adv. Neural Info Proc. Sys. $\underline{\&}$, pp. 931-937, 1996.

[5] Cornelis Stam, M. Brekspear, A-M van Capellen van Walsum, B W. van Dijk, "NL synchronization in F-EEG and whole-head MEG recording of healthy subjects,” Hum. Brain MappingV.19, pp.63-78, 2003.

[6] G. Tononi, G.M. Edelman, "A measure for brain complexity: relating functional segregation and integration in the nervous system”, Proc. Natl. Acad. Sci. USA V.91, pp5033-5037, 1994.

[7] W. Singer, "Consciousness and the binding problem," Ann NY Acad. Sci. V.929, pp.123-146, 2001

[8] W.J. Freeman, L.J. Rogers, "Fine temporal resolution of analytic phase reveals episodic synchronization by state transitions in gamma F-EEGs,” J. Neurophysiol V.97,pp.937-945, 2002.

[9] J.J. Hopfield, C.D. Brody, "What is a moment? Transient synchrony as a collective mechanism for spatiotemporal integration," Proc. Natl. Acad. Sci. USA V98:pp1282-1287, 2001.

[10] Sabine J. Regel, Gilberte Tinguely, Ju Rgen Schuderer, Martin Adam, Niels Kuster, Hans-Peter Landolt, Peter Achermann, "Pulsed radio-frequency electromagnetic fields: dose-dependent effects on sleep, the sleep F-EEG and cognitive performance," J. Sleep Res. (2007) 16, 253-258.

[11] Benedek G,Obál F Jr, Lelkes Z,Obál F "Thermal and chemical stimulations of the hypothalamic heat detectors: the effects of the F-EEG."Acta Physiologica Academiae Scientiarum Hungaricae[1982, 60(1-2):27-35]

[12] Tom Deboer, "Brain temperature dependent changes in the electroencephalogram power spectrum of humans and animals," J. Sleep Res. (1998) 7, 254-262

[13] Fadi Karameh, Munther Dahleh, “Automated classification of F-EEG signals in brain tumor diagnostics,” Proc. Am. Control Conf. June 2000. pp. 4169-4173. 
[14] J. Gardiner, R. Overall, J. Marc, "The fractal nature of the brain: F-EEG data suggests that the brain functions as a "quantum computer" in 5 8 dimensions," NeuroQuantology $\underline{\mathrm{V} 8}$, pp137-141, 2010.

[15] F.C. Morabito, D. Labate, A. Bramanti, F. La Foresta, G. Morabito, I. Palamara, H. Szu, "Enhanced compressibility of F-EEG signal in Alzheimer's disease Patients," IEEE Sensors, 2013.

[16] J. Jenkins, Q. Nguyen, J. Reynolds, W. Horner, and H. Szu, The Physiology of Keystroke Dynamics, in Independent Component Analyses, Wavelets, Neural Networks, Biosystems, and Nanoengineering IX, 2011

[17] H. Szu, C. Hsu, J. Jenkins, J. Willey, and J. Landa, Capturing Significant Events with Neural Networks. Neural Networks, 29-30 (May 2012): 1-7.

[18] J. Jenkins and H. Szu, Recognizing foreground-background interaction, in Independent Component Analyses, Wavelets, Neural Networks, Biosystems, and Nanoengineering VIII, 2010

[19] Harold Szu, Charles Hsu, Gyu Moon, Takeshi Yamakawa, Binh Tran, Tzyy Ping Jung and Joseph Landa, "Smartphone Household Wireless, Electroencephalogram Hat,” Applied Comp. Intell. And Soft Computing, Vol. 2013, pp. 1-8, 2013.

[20] C. Gross, "Genealogy of the 'Grandmother Cell", The Neuroscientist, pp. 84-90, 2002 